\title{
Disease-Modifying Therapies and Coronavirus Disease 2019 Severity in Multiple Sclerosis
}

\author{
Maria P. Sormani, PhD (10, 1,2 Nicola De Rossi, MD, ${ }^{3}$ Irene Schiavetti, PhD, ${ }^{1}$ \\ Luca Carmisciano, MD, ${ }^{1}$ Cinzia Cordioli, MD, ${ }^{3}$ Lucia Moiola, MD, ${ }^{4}$ Marta Radaelli, MD, ${ }^{5}$ \\ Paolo Immovilli, MD, ${ }^{6}$ Marco Capobianco, MD, ${ }^{7}$ Maria Trojano, MD, ${ }^{8}$ Paola Zaratin, PhD, 9 \\ Gioacchino Tedeschi, MD, ${ }^{10}$ Giancarlo Comi, MD (1), ${ }^{11}$ Mario A. Battaglia, MD, 9,12 \\ Francesco Patti, MD (1), ${ }^{13,14}$ Marco Salvetti, MD, ${ }^{15,16}$ and the Musc-19 Study Group
}

Objective: This study was undertaken to assess the impact of immunosuppressive and immunomodulatory therapies on the severity of coronavirus disease 2019 (COVID-19) in people with multiple sclerosis (PwMS).

Methods: We retrospectively collected data of PwMS with suspected or confirmed COVID-19. All the patients had complete follow-up to death or recovery. Severe COVID-19 was defined by a 3-level variable: mild disease not requiring hospitalization versus pneumonia or hospitalization versus intensive care unit (ICU) admission or death. We evaluated baseline characteristics and MS therapies associated with severe COVID-19 by multivariate and propensity score (PS)-weighted ordinal logistic models. Sensitivity analyses were run to confirm the results.

Results: Of 844 PwMS with suspected $(n=565)$ or confirmed $(n=279)$ COVID-19, 13 (1.54\%) died; 11 of them were in a progressive MS phase, and 8 were without any therapy. Thirty-eight (4.5\%) were admitted to an ICU; $99(11.7 \%)$ had radiologically documented pneumonia; 96 (11.4\%) were hospitalized.

After adjusting for region, age, sex, progressive MS course, Expanded Disability Status Scale, disease duration, body mass index, comorbidities, and recent methylprednisolone use, therapy with an anti-CD20 agent (ocrelizumab or rituximab) was significantly associated (odds ratio $[\mathrm{OR}]=2.37,95 \%$ confidence interval $[\mathrm{Cl}]=1.18-4.74, p=0.015$ ) with increased risk of severe COVID-19. Recent use ( $<1$ month) of methylprednisolone was also associated with a worse outcome $(\mathrm{OR}=5.24,95 \% \mathrm{Cl}=2.20-12.53, p=0.001)$. Results were confirmed by the PS-weighted analysis and by all the sensitivity analyses.

Interpretation: This study showed an acceptable level of safety of therapies with a broad array of mechanisms of action. However, some specific elements of risk emerged. These will need to be considered while the COVID-19 pandemic persists.

ANN NEUROL 2021;89:780-789

View this article online at wileyonlinelibrary.com. DOI: 10.1002/ana.26028

Received Nov 11, 2020, and in revised form Jan 18, 2021. Accepted for publication Jan 18, 2021.

Address correspondence to Dr Salvetti, Dipartimento di Neuroscienze, Salute Mentale e Organi di Senso, AOU S. Andrea, via di Grottarossa 1035-1039, 00189-Rome, Italy. E-mail: marco.salvetti@uniroma1.it

The members of the Musc-19 Study Group are identified in a supplementary table.

From the ${ }^{1}$ Department of Health Sciences, University of Genoa, Genoa, Italy; ${ }^{2}$ IRCCS Ospedale Policlinico San Martino, Genoa, Italy; ${ }^{3}$ Centro Sclerosi Multipla ASST Spedali Civili di Brescia, Montichiari, Italy; ${ }^{4}$ Department of Neurology, Multiple Sclerosis Center, IRCCS Ospedale San Raffaele, Milan, Italy; ${ }^{5}$ Department of Neurology and Multiple Sclerosis Center, ASST Papa Giovanni XXIII, Bergamo, Italy; ${ }^{6}$ Multiple Sclerosis Center, Ospedale Guglielmo da Saliceto, Piacenza, Italy; ${ }^{7}$ Department of Neurology, Regional Referral Multiple Sclerosis Center, University Hospital San Luigi, Orbassano, Torino, Italy; ${ }^{8}$ Department of Basic Medical Sciences, Neurosciences, and Sense Organs, University of Bari, Bari, Italy; ${ }^{9}$ Research Department, Italian Multiple Sclerosis Foundation, Genoa, Italy; ${ }^{10}$ Department of Advanced Medical and Surgical Sciences, University of Campania, Naples, Italy; ${ }^{11}$ Institute of Experimental Neurology, IRCCS Ospedale San Raffaele, Milan, Italy; ${ }^{12}$ Department of Life Sciences, University of Siena, Siena, Italy; ${ }^{13}$ Department of Medical and

Surgical Sciences and Advanced Technologies, GF Ingrassia, University of Catania, Catania, Italy; ${ }^{14}$ Centro Sclerosi Multipla, Policlinico Catania, University of Catania, Catania, Italy; ${ }^{15}$ Department of Neuroscience, Mental Health, and Sensory Organs, Sapienza University of Rome, Rome, Italy; and ${ }^{16}$ Unit of Neurology, IRCCS Neuromed, Pozzilli, Italy 
$\mathrm{T}$ he COVID-19 pandemic and the many questions about the postpandemic period complicate the management of patients who need therapies that impact on the immune system. Data available so far are overall reassuring, excluding major safety issues. ${ }^{1-4}$ However, the robustness of results is not optimal due to the relatively small samples, collected in a situation of urgency. Furthermore, for most of the autoimmune diseases, many possible therapies are available, thus increasing the heterogeneity of the datasets. It is therefore necessary to rely on larger and better characterized case series to improve data quality and relevance. The results will inform clinical decisions that will have a long-term impact, given the chronicity of the diseases, the duration of therapies, and the long-lasting effects of some treatments.

Multiple sclerosis (MS) is the major cause of neurological disability in young adults, with approximately 2.3 million people affected worldwide. ${ }^{5} \mathrm{Up}$ to $70 \%$ of people with MS (PwMS) are treated with disease-modifying therapies (DMTs) that impact on the immune response and may carry an increased probability of infection. ${ }^{6}$ This risk must be balanced against the consequences of poorly controlled MS. Hence, reliably assessing the risk of coronavirus disease 2019 (COVID-19) in these patients is an important public health issue, and more data are needed to guide clinical practice, as pointed out in recent reviews. ${ }^{7}$ So far, the largest study based on data is the paper on the French cohort, ${ }^{3}$ but the sample size did not allow drawing conclusions about the association of DMTs and COVID-19 severity.

We present the results of an observational study on PwMS with a confirmed or suspected COVID-19 infection, based on clinician-reported data. The study was conducted in Italy, a high-prevalence area for $\mathrm{MS}^{8}$ that was the first European country to suffer the effects of the pandemic. The results are presented after the preplanned sample size needed to reach conclusions on the effect of DMTs on COVID-19 severity was reached.

\section{Patients and Methods}

\section{Data Sources}

We obtained clinician-reported demographic and clinical data on PwMS with a confirmed or suspected COVID-19 infection from 85 Italian MS centers (Supplementary Table S2). We used a common web-based electronic case report form (eCRF) to collect the data and a unified protocol to analyze them. Data were obtained after datasharing agreements between the Italian MS Society, the Italian Neurological Society, the University of Genoa, and all the MS centers involved. Demographic, MS history, COVID-19 infection, and follow-up data were collected.
The study was approved by the regional ethics committee of Liguria (University of Genoa; n 130/2020-DB id 10433) and at a national level by the Italian Medicines Agency.

\section{Study Population}

We included adult MS patients who had been in contact with their neurologist because of a confirmed or suspected infection by severe acute respiratory syndrome coronavirus 2 (SARS-CoV-2) during the observation period (January 15 to September 10, 2020). The confirmed cases were those with a positive test (reverse transcriptase polymerase chain reaction on nasal and pharyngeal swabs) for SARSCoV-2 or a positive serological test obtained at any point during the observation period; the suspected cases were those who had radiological findings or symptoms highly suggestive of SARS-CoV-2 infection, according to medical judgment (cough, fever, shortness of breath, sudden onset of anosmia, ageusia, dysgeusia), and/or had close contact with a confirmed COVID-19 case in the 14 days prior to the onset of symptoms. Data were collected retrospectively from the first contact until an outcome (death or recovery), taking as baseline the day of symptoms appearance. The first contact was a hospital visit, a phone call, or a web-based visit, upon patients' or clinicians' request. Clinicians collected data by reviewing patients' clinical charts.

\section{Variables Assessed}

Collected data are detailed in the eCRF. We collected demographic data, including age, sex, body mass index (BMI), patient-reported race and ethnic group, work status, number of cohabitants and number of cohabitants positive for SARS-CoV-2 infection, patient-reported smoking and drinking status, and comorbidities; MS history data, including type of MS (relapsing-remitting MS [RRMS], primary progressive, or secondary progressive), disease onset date, Expanded Disability Status Scale (EDSS), DMTs at the date of symptoms start, and date of last treatment dose; and COVID-19 infection data, including suspected geographic area of infection, symptoms, laboratory and radiological data, hospitalization, pneumonia and severity of pneumonia according to radiological examinations and ventilation support, therapies for COVID-19, intensive care unit (ICU) admission, and recovery or death.

Each week a query for follow up update was sent to all centers, until they filled the outcome report (death or recovery).

\section{End Points}

We run 2 main analyses on the following primary endpoints: (1) observed number of deaths over the whole 
follow-up; and (2) severe COVID-19 characterized by a 3-level variable; the highest severity level was death or ICU admission, the intermediate severity level was diagnosis of pneumonia or hospitalization, and the lowest severity level was a milder disease with no need for hospitalization or documented diagnosis of pneumonia.

\section{Statistical Analysis}

The web-based data collection started on March 1, 2020 and closed for the analysis on September 10, 2020. The first date of symptom appearance retrospectively reported was January 15, 2020. All the patients had complete follow-up to death or recovery.

For the sample size calculations, we determined that 800 patients were needed, assuming a rate of severe outcomes (death, ICU admission, pneumonia, or hospitalization) of $20 \%$ in the reference group and a comparison group made of $15 \%$ of the full cohort, to have a power of $90 \%$ to detect an increased risk of severe outcome in the comparison group quantified by an odds ratio $(\mathrm{OR})=2.0$ at a confidence level of 0.05 .

To make efficient use of the available data, we used multiple imputation of missing values for missing baseline data. Imputation was performed using chained equations, where each incomplete variable is imputed by a separate model and implemented trough the "mice" $\mathrm{R}$ package. Continuous variables (age, height, weight, BMI, and disease duration) were parameterized as numeric data and imputed with the predictive mean matching method, whereas polytomous logistic regression was used for the unordered categorical variables (such as MS phenotype). EDSS and disease duration were used to impute the MS phenotype; age and MS phenotype were used to impute disease duration; age, height, or weight was used to impute BMI.

To assess risk factors at symptoms onset for a severe disease course, we ran a multivariate ordinal logistic regression assuming proportional ORs with severe outcome (defined as a 3-level factor) as the dependent variable, after assessing that the assumptions of proportional odds were satisfied. The ORs reflect the multiplicative change in the odds of being at a higher level of the dependent variable for every one-unit increase of the independent variable. The multivariate model included age, sex, BMI, EDSS, disease duration, MS disease phase (RRMS vs progressive), presence of comorbidities, methylprednisolone use within 1 month since COVID-19 symptoms onset, and DMT class as independent variables, stratified by macroregion (defined as Lombardy; Northern Italy, including Piedmont, Veneto, Emilia-Romagna, and Liguria; and the rest of Italy). DMT class was coded as an 8-level variable as no therapy, interferon, glatiramer-acetate, teriflunomide, dimethyl fumarate, natalizumab, anti-CD20 (rituximab or ocrelizumab), or other. Alemtuzumab and cladribine were grouped in the "other" group, because the number of patients in these two therapeutic arms was too low to draw meaningful conclusions. "No therapy" was used as the reference category. We also ran the same ordinal logistic analysis after a multinomial propensity score (PS) weighting, as an alternative method to balance the differences of baseline characteristics among patients treated with different DMTs. The weights were calculated using an iterative robust approach based on the Generalized Boosted Model (GBM). ${ }^{10}$ The balance among treatment groups was defined by the standardized mean difference, and the optimal GBM iteration was found by minimizing this quantity.

Results were also presented taking dimethyl fumarate, which is the most frequently used therapy in Italy, as the reference therapy group (Supplementary Appendix). Additional analyses were focused on the anti-CD20 therapies using the same multivariate ordinal logistic model adjusted for all the baseline variables; we investigated the effect of being on anti-CD20 versus not being on anti$\mathrm{CD} 20$, the effect of the distance from last infusion (0-3 months, $>3$ months), and the effect of the time since therapy start ( $<6$ months, 6-12 months, $>12$ months).

Sensitivity analyses were run by repeating all the analyses on the subgroup of confirmed cases only, in the subgroup of patients with a RRMS course, in a model including only patients with complete baseline data with no imputation and using a leave one out procedure rerunning the analysis excluding one of the 3 major centers (Brescia, Bergamo, Milan) at a time (Supplementary Appendix).

\section{Role of the Funding Source}

Roche donated the web-based platform for data collection. Roche did not have any role in study design; in analysis and interpretation of data; in the writing of the report; or in the decision to submit the paper for publication.

\section{Results}

\section{Patients Characteristics}

On September 10, 844 PwMS with complete follow-up from first contact to the outcome (recovery or death) from 85 centers were included in the dataset (Supplementary Appendix). Thirty-four percent (286/844) of patients were from 3 centers in Lombardy (ASST Spedali Civili, Brescia-Montichiari; Papa Giovanni XXIII Hospital, Bergamo; and San Raffaele Hospital, Milan); the distribution of patients by region is shown in Supplementary Table S3. 


\section{TABLE 1. Baseline Demographic and Clinical Characteristics of Included Patients}

\section{Characteristic}

Age, n (\%)

$<40 \mathrm{yr}$
$40-59 \mathrm{yr}$
$60-79 \mathrm{yr}$
$\geq 80 \mathrm{yr}$

Female sex, n (\%)

BMI, mean (SD)

Comorbidities, n (\%)

MS phenotype, n (\%)

Primary progressive

Relapsing-remitting

Secondary progressive

Missing data

MS disease duration,

median (IQR)

EDSS, median (IQR)

MS treatment, n (\%)

\begin{tabular}{|c|c|c|c|}
\hline Dimethyl fumarate & $174(20.6)$ & $131(23.2)$ & $43(15.4)$ \\
\hline Fingolimod & $94(11.1)$ & $59(10.4)$ & $35(12.5)$ \\
\hline Ocrelizumab & $89(10.5)$ & $58(10.3)$ & $31(11.1)$ \\
\hline Natalizumab & $85(10.1)$ & $51(9.0)$ & $34(12.2)$ \\
\hline Interferon & $73(8.6)$ & $52(9.2)$ & $21(7.5)$ \\
\hline Copaxone & $70(8.3)$ & $52(9.2)$ & $18(6.5)$ \\
\hline Teriflunomide & $64(7.6)$ & $37(6.5)$ & $27(9.7)$ \\
\hline Alemtuzumab & $14(1.7)$ & $11(1.9)$ & $3(1.1)$ \\
\hline Cladribine & $11(1.3)$ & $7(1.2)$ & $4(1.4)$ \\
\hline Azathioprine & $10(1.2)$ & $4(0.7)$ & $6(2.2)$ \\
\hline Rituximab & $5(0.6)$ & $4(0.7)$ & $1(0.4)$ \\
\hline Methotrexate & $1(0.1)$ & $1(0.2)$ & $0(0.0)$ \\
\hline Mitoxantrone & $1(0.1)$ & $1(0.2)$ & $0(0.0)$ \\
\hline Other & $2(0.2)$ & $2(0.4)$ & $0(0.0)$ \\
\hline None & $151(17.9)$ & $95(16.8)$ & $56(20.1)$ \\
\hline $\begin{array}{l}\text { ious methylprednisolone, } \\
\text { (0) }\end{array}$ & $26(3.1)$ & $16(2.8)$ & $10(3.6)$ \\
\hline
\end{tabular}

$\begin{array}{ccc}279(33.1) & 203(35.9) & 76(27.2) \\ 471(55.8) & 312(55.2) & 159(57.0) \\ 92(10.9) & 50(8.8) & 42(15.1) \\ 2(0.2) & 0(0.0) & 2(0.7) \\ 593(70.3) & 410(72.6) & 183(65.6) \\ 23.82(4.37) & 23.61(4.37) & 24.28(4.37) \\ 188(22.3) & 115(20.4) & 73(26.2)\end{array}$

$44(5.2)$

$676(80.1)$

$91(10.8)$

$33(3.9)$

$10.2(4.7-17.1)$
Suspected, $n=565$

$$
24(4.2)
$$$$
20(7.2)
$$$$
469(83.0)
$$$$
46(8.1)
$$

$9.6(4.3-16.2)$

$2(1.50-3.50)$

$2(1.50-4)$ 207 (74.2)

45 (16.1)

7 (2.5)$$
26 \text { (4.6) }
$$

Confirmed, $\mathbf{n}=279$

$11.8(5.4-18.5)$

$(1.50$


Table 1 reports the baseline demographic and clinical characteristics of the cohort. The mean age was 45 years (range $=18-82$ ), the percentage of females was $70.3 \%$, the median EDSS was 2 (interquartile range [IQR] $=1.50-4)$, and the proportion of progressive patients was $16 \%$. Six hundred ninety-three (82\%) patients were treated with a DMT at the time of COVID-19 presumed symptoms onset.

Three hundred eighty-four patients $(45.5 \%)$ had at least one laboratory test executed for the COVID-19 diagnosis; 307 (36.4\%) patients were tested in the active phase with a swab and $127(15.0 \%)$ had a serological test after recovery. Overall, 279 (33.1\%) were classified as confirmed cases. Because the test during the pandemic peak was done only in the most severe patients, confirmed cases are expected to be more severe than suspected cases. In the confirmed cases subgroup, there was a higher proportion of older subjects than in the suspected cases subgroup ( $>60$ years $=16 \%$ vs $9 \%)$ and more patients in the progressive MS phase (23\% vs $12 \%)$.

The baseline characteristics of the cohort according to DMT received are reported in Table 2; patients with no therapy were older (mean age $=53.4$ years), with a higher EDSS (mean EDSS $=5.5$ ) and with a higher proportion of progressive patients (45.7\%) than patients treated with DMTs. Among treated patients, those treated with interferon, glatiramer-acetate, or teriflunomide were older than those in other DMT groups, and patients treated with anti-CD20 had a higher EDSS (mean EDSS $=3$ ) and a larger portion of progressive patients $(25.6 \%)$ than those treated with other DMTs, but not larger than the untreated group.

Thirteen (1.54\%) patients died, and their characteristics are described in Table 3; 8 of them were PwMS with no therapy, all in a progressive disease phase (1 primary progressive MS, 7 secondary progressive MS). Five of them were treated (rituximab, ocrelizumab, natalizumab, glatiramer-acetate, dimethyl fumarate), and only 2 of them were in a RRMS phase (glatiramer-acetate and natalizumab). The mean time from symptoms to death was 18 days (range $=5-54$ days).

\section{Risk Factors for a Severe COVID-19 Outcome}

Thirty-eight (4.5\%) patients were admitted to an ICU (7 of them died); 99 cases (11.7\%) of radiologically documented pneumonia were reported; 96 patients $(11.4 \%)$ were hospitalized. Forty-four patients $(5.2 \%)$ were in the highest severity class (death or ICU admission), and 92 patients (10.9\%) were in the intermediate severity class (pneumonia or hospitalization). Twenty-six patients (3.1\%) received methylprednisolone in the month preceding the first symptoms of COVID-19.
Risk factors for severe COVID-19 in univariate, multivariate, and PS-weighted analysis are reported in Table 4. The assumptions of proportional odds were satisfied. Older age, male sex, higher EDSS, longer MS duration, presence of comorbidities, and progressive MS course were all risk factors for a more severe disease in univariate analysis. Methylprednisolone use within 1 month before COVID19 symptoms onset was associated with increased risk $(\mathrm{OR}=3.38,95 \%$ confidence interval $[\mathrm{CI}]=1.49-7.67$, $p=0.004)$. Taking no therapy as the reference category indicated a reduced risk for all the DMTs, with OR ranging from 0.34 to 0.50 , except for the anti-CD20 therapies (ocrelizumab/rituximab, $\mathrm{OR}=0.94$ ). When adjusting the OR in the multivariate model, the risk factors with a significant impact on COVID-19 severity were age, sex, EDSS, recent use of methylprednisolone, and anti-CD20 therapy. Age and EDSS fully explained the apparent risk associated with no therapy as compared to the other DMTs, and the only treatment associated with a higher risk of a severe COVID-19 disease was anti-CD20 therapy (OR $=2.37$, $95 \% \mathrm{CI}=1.18-4.74, p=0.015)$. Also, the increased risk associated with the use of recent methylprednisolone was confirmed $(\mathrm{OR}=5.24,95 \% \mathrm{CI}=2.20-12.53, p=0.001)$. The PS-weighted characteristics of patients according to the different DMTs received are reported in Supplementary Table S11. The DMT effects on COVID-19 severity are replicated by the PS-weighted analysis. The OR for antiCD20 therapy was $3.91(95 \% \mathrm{CI}=1.71-8.91, p=0.001)$.

The results taking dimethyl fumarate as the reference category are reported in the Supplementary Appendix and are in line with the above results. All the sensitivity analyses confirmed these findings; the anti-CD20-treated patients had an $\mathrm{OR}=2.62(p=0.05)$ in the subgroup of confirmed cases, an $\mathrm{OR}=2.87(p=0.03)$ in the subgroup of RRMS patients, and an OR $=2.69(p=0.026)$ using only complete baseline data with no imputation (Supplementary Appendix).

A further result that deserves attention is the risk reduction observed in patients treated with interferon $(\mathrm{OR}=0.67)$; however, this does not reach a statistical significance. Among the 73 patients on interferon, no ICU admissions or deaths were recorded.

To give an idea of the absolute risk of severe COVID-19 according to DMT, Figure reports the percentage of patients with severe events (pneumonia, hospitalization, ICU, or death) in the group treated with anti$\mathrm{CD} 20$, in the untreated group, and in the group treated with other DMTs. To adjust these values for the confounding factors, the figure was restricted to patients $<65$ years old and with EDSS $<6.5(n=733)$, and presented separately for the RRMS $(n=675)$ and progressive MS ( $\mathrm{n}=58)$ groups. The percentage of subjects with 


\begin{tabular}{|c|c|c|c|c|c|c|c|c|c|}
\hline Characteristic & $\begin{array}{l}\text { No } \\
\text { Therapy, } \\
n=151\end{array}$ & IFN, $\mathbf{n}=73$ & GA, $n=70$ & $\begin{array}{l}\text { Teri, } \\
\mathrm{n}=\mathbf{6 4}\end{array}$ & $\begin{array}{l}\text { DMF, } \\
\mathrm{n}=174\end{array}$ & $\begin{array}{l}\text { FTY, } \\
\mathrm{n}=94\end{array}$ & $\begin{array}{l}\text { Nat, } \\
\mathrm{n}=85\end{array}$ & $\begin{array}{l}\text { Anti- } \\
\text { CD20, } \\
\mathbf{n}=94^{\mathrm{a}}\end{array}$ & $\begin{array}{l}\text { Other, } \\
\mathrm{n}=39\end{array}$ \\
\hline Age, mean (SD) & 53.4 (13.4) & $47.5(10.5)$ & $45.7(11.3)$ & $49.1(9.4)$ & $41.4(10.9)$ & $44.2(9.5)$ & $37.9(9.8)$ & $42.0(10.0)$ & $42.2(13.8)$ \\
\hline Female sex, n (\%) & $104(68.9)$ & $53(72.6)$ & $48(68.6)$ & $44(68.8)$ & $114(65.5)$ & $74(78.7)$ & $64(75.3)$ & $64(68.1)$ & $28(71.8)$ \\
\hline BMI, mean (SD) & $24.6(4.6)$ & $24.3(2.7)$ & $25.2(5.0)$ & $24.7(4.5)$ & $23.3(4.1)$ & $23.9(5.2)$ & $22.7(4.1)$ & $22.6(3.7)$ & $23.2(3.8)$ \\
\hline \multicolumn{10}{|l|}{$\begin{array}{l}\text { MS phenotype, } \\
\text { n (\%) }\end{array}$} \\
\hline Primary progressive & $22(14.6)$ & $0(0.0)$ & $1(1.4)$ & $0(0.0)$ & $0(0.0)$ & $2(2.1)$ & $1(1.2)$ & $16(17.0)$ & $2(5.1)$ \\
\hline Relapsing-remitting & $65(43.0)$ & $65(89.0)$ & $64(91.4)$ & $59(92.2)$ & $165(94.8)$ & $81(86.2)$ & $83(97.6)$ & $67(71.3)$ & $27(69.2)$ \\
\hline Secondary progressive & $47(31.1)$ & $6(8.2)$ & $2(2.9)$ & $3(4.7)$ & $5(2.9)$ & $9(9.6)$ & $0(0.0)$ & $9(9.6)$ & $10(25.6)$ \\
\hline Missing data & $17(11.3)$ & $2(2.7)$ & $3(4.3)$ & $2(3.1)$ & $4(2.3)$ & $2(2.1)$ & $1(1.2)$ & $2(2.1)$ & $0(0.0)$ \\
\hline $\begin{array}{l}\text { MS disease duration, } \\
\text { median yr (IQR) }\end{array}$ & $16.2(8-23)$ & $12.0(6-19)$ & $10.1(5-17)$ & $11.4(6-15)$ & $5.5(3-13)$ & $11.8(7-18)$ & $9.1(5-15)$ & $8.2(4-13)$ & $12.9(5-18)$ \\
\hline EDSS, median (IQR) & $5.5(2-7)$ & $1.5(1-2.5)$ & $1.5(1-2)$ & $2.0(1.5-3)$ & $1.5(1-2)$ & $2.0(1.5-3)$ & $2.0(1.5-3)$ & $3.0(1.5-4.5)$ & $3.0(1.5-6)$ \\
\hline Methylprednisolone, ${ }^{\mathrm{b}} \mathrm{n}$ & $9(6.0)$ & $2(2.7)$ & $1(1.4)$ & $2(3.1)$ & $4(2.3)$ & $4(4.3)$ & $1(1.2)$ & $1(1.1)$ & $2(5.1)$ \\
\hline
\end{tabular}

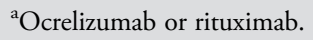

${ }^{\mathrm{b}}$ One month before symptoms onset.

BMI = body mass index; DMF = dimethyl fumarate; EDSS = Expanded Disability Status Scale; FTY = fingolimod; GA = glatiramer-acetate; IFN = interferon; IQR = interquartile range; $\mathrm{MS}$ = multiple sclerosis; Nat = natalizumab; $\mathrm{SD}=$ standard deviation; Teri = teriflunomide.

\section{TABLE 3. Characteristics of Deceased Patients}

\begin{tabular}{|c|c|c|c|c|c|}
\hline Sex, Age, yr & $\begin{array}{l}\text { Disease } \\
\text { Duration }\end{array}$ & $\begin{array}{l}\text { Disease } \\
\text { Phase }\end{array}$ & EDSS & Therapy & Comorbidities \\
\hline M, 63 & 33 & SPMS & 7 & No therapy & Diabetes \\
\hline M, 67 & 2 & PPMS & 7.5 & No therapy & CHD, hypertension, HBV \\
\hline M, 68 & 21 & SPMS & 6 & $\begin{array}{l}\text { Dimethyl } \\
\text { fumarate }\end{array}$ & $\begin{array}{l}\text { Cerebrovascular disease, hypertension, depression, } \\
\text { TBC }\end{array}$ \\
\hline $\mathrm{F}, 57$ & 20 & SPMS & 9 & No therapy & No \\
\hline M, 76 & 17 & SPMS & 6.5 & No therapy & CHD, hypertension, depression, dyslipidemia \\
\hline $\mathrm{F}, 52$ & 1 & RRMS & 5 & Natalizumab & No \\
\hline $\mathrm{F}, 50$ & 27 & SPMS & 6 & Ocrelizumab & No \\
\hline M, 59 & 33 & SPMS & 9 & No therapy & No \\
\hline F, 68 & 19 & SPMS & 5.5 & No therapy & No \\
\hline $\mathrm{F}, 54$ & 20 & SPMS & 7 & Rituximab & No \\
\hline M, 64 & 10 & RRMS & 2 & $\begin{array}{l}\text { Glatiramer- } \\
\text { acetate }\end{array}$ & No \\
\hline M, 63 & 33 & SPMS & 6.5 & No therapy & Diabetes, bipolar disorder \\
\hline M, 60 & 30 & SPMS & 9 & No therapy & Hypertension, cerebrovascular disease, CHD \\
\hline
\end{tabular}




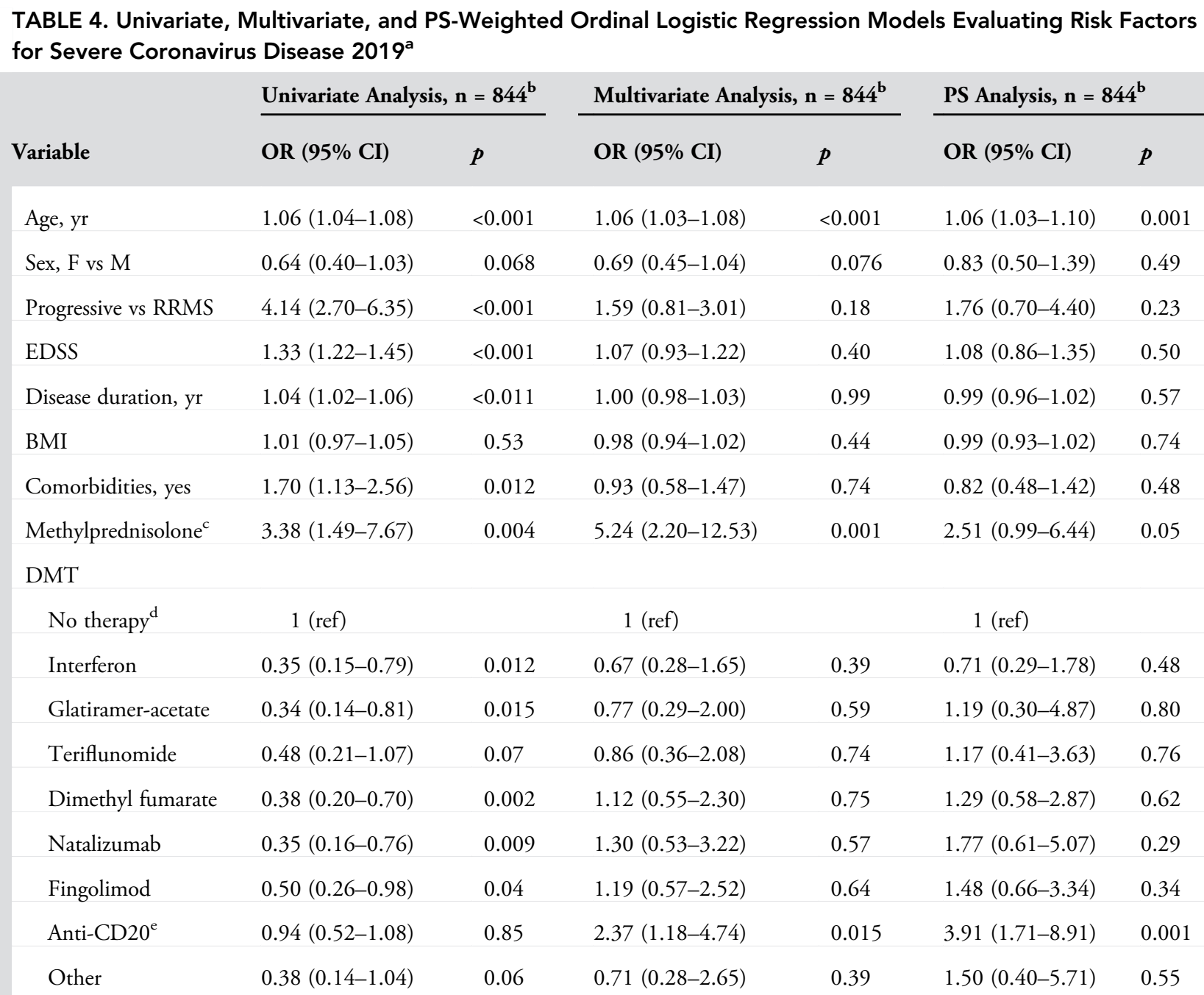

${ }^{\mathrm{a}}$ Intensive care unit or death vs hospitalization or pneumonia vs milder symptoms not requiring hospitalization and no documented pneumonia.

${ }^{\mathrm{b}}$ All the analyses are adjusted for macroregion (Lombardy; Northern Italy, including Veneto, Emilia-Romagna, Piedmont, and Liguria; and the rest of Italy).

${ }^{c}$ One month before symptoms onset.

${ }^{\mathrm{d}}$ No therapy was chosen as the reference class.

${ }^{\mathrm{e}}$ Ocrelizumab or rituximab.

$\mathrm{BMI}=$ body mass index; $\mathrm{CI}=$ confidence interval; DMT = disease-modifying therapy; EDSS = Expanded Disability Status Scale; $\mathrm{F}=$ female; $\mathrm{M}=$ male; OR = odds ratio; PS = propensity score; RRMS = relapsing-remitting multiple sclerosis.

severe events was higher in the group of patients treated with anti-CD20 both in the RRMS and in the progressive MS patients; in the RRMS group, the proportion of patients deceased or admitted to ICU in the anti-CD20 group was $6.1 \%$, versus $4.2 \%$ in the untreated and $2.2 \%$ in the other DMTs group. In the progressive MS group, it was $12.5 \%$ in the anti-CD20 group, $10.5 \%$ in the untreated group, and $4.3 \%$ in the other DMTs group. The percentage of patients with an intermediate severity (pneumonia or hospitalization) had the same trend of increase in the anti-CD20 group.

The increase of risk associated with anti-CD20 was maintained on hard end points (death or ICU admission), even if the small number of events did not allow this to reach statistical significance (Supplementary Appendix).

The adjusted OR for anti-CD20 therapy versus all the other therapies was $2.37(95 \% \mathrm{CI}=1.36-4.12$, $p=0.002)$. Exploratory analyses revealed no association between COVID-19 severity and the time passed since the last anti-CD20 infusion $(\mathrm{OR}=2.77,95 \% \mathrm{CI}=1.31-$ 5.89, $p=0.012$ for last infusion within 3 months and $\mathrm{OR}=2.05,95 \% \mathrm{CI}=0.97-4.28, p=0.023$ for last infusion before 3 months), whereas there was a trend with therapy duration; as compared to PwMS treated with other therapies, patients on anti-CD20 therapy for $<6$ months had an $\mathrm{OR}=1.65(95 \% \mathrm{CI}=0.56-4.90, p=0.36)$, 


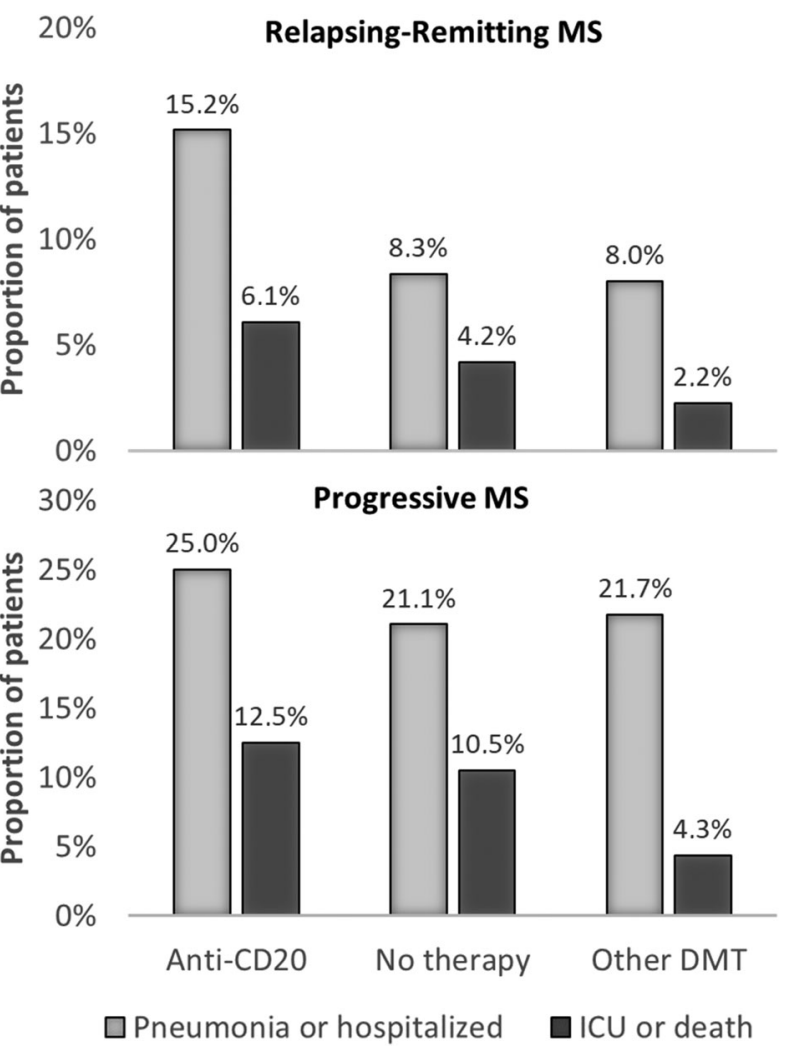

FIGURE: Percentage of patients with pneumonia/ hospitalization and intensive care unit (ICU)/death in the subgroup of subjects with age $<65$ years and Expanded Disability Status Scale $<6.5$, according to multiple sclerosis (MS) phenotype (relapsing-remitting vs progressive MS), in the anti-CD20, no therapy, and other therapies groups. DMT $=$ disease-modifying therapy.

patients on anti-CD20 therapy for 6-12 months had an $\mathrm{OR}=2.24(95 \% \mathrm{CI}=0.91-5.55, p=0.08)$, and patients on anti-CD20 therapy for $>12$ months had an OR $=2.98$ $(95 \%$ CI $=1.37-6.46, p=0.006 ;$ Supplementary Appendix).

No severe outcomes (no pneumonia, hospitalization, ICU, or death) were observed in the 11 patients treated with cladribine or in the 14 patients treated with alemtuzumab.

\section{Discussion}

This study shows an acceptable level of safety of immunomodulatory and immunosuppressive therapies in MS during the COVID-19 pandemic. However, two results deserve attention.

First, 11 of 13 deaths occurred in persons with advanced disease and disability (median EDSS $=7$ ). The median age of people who died was 63 years versus a median age of 45 years for those who recovered. Higher EDSS was associated in multivariate analysis with COVID-19 severity also in the French MS population. ${ }^{3}$ These findings strengthen the need to enforce prevention strategies for people with advanced disability and older age during the pandemic. ${ }^{11}$

Second, we observed an increased frequency of a severe COVID-19 in people treated with anti-CD20 therapies compared to other DMTs. Smaller case series suggested an increased susceptibility to COVID-19 in PwMS taking B-cell-depleting monoclonal antibodies, ${ }^{12-14}$ without major effects on the severity of COVID-19. ${ }^{13-15}$ In the largest study published so far (the French study), DMT exposure was not included in the multivariate model. The results of the univariate analysis are very similar to those presented here. ${ }^{3}$ It will be informative to evaluate the OR for the impact of DMTs on severity in the French cohort in the multivariate model, to check the size of effect, irrespective of the statistical significance. Data are available about the general risk of infections during DMTs for MS; a nationwide Swedish cohort examined the infection risks in PwMS treated with DMTs. ${ }^{16}$ In that study, rituximab was associated with the highest rate of serious infections. However, the use of herpes antivirals was lower with rituximab compared to fingolimod and natalizumab; ocrelizumab clinical trials reported an increased risk of respiratory tract infections, ${ }^{17,18}$ and safety issues emerged in diseases other than MS. ${ }^{19}$ With respect to the association with treatment duration, it has been shown that long-term anti-CD20 treatment is associated with the risk of hypogammaglobulinemia in neuromyelitis optica-spectrum disorder. ${ }^{20} \mathrm{~A}$ protracted treatment may impact on the protective, anti-SARS-CoV-2 humoral response ${ }^{21}$ and on a pre-existing humoral and cellular immune repertoire, recently described in unexposed individuals. ${ }^{22,23}$

We did not observe a link between time to last infusion of ocrelizumab and COVID-19 risk. This preliminary result needs to be confirmed, but it is consistent with the idea that the immunological effects of ocrelizumab may last longer than 6 months. Reducing the frequency of dosing, or adjusting it according to the monitoring of Bcell repopulation kinetics in individual patients, ${ }^{20}$ may maintain efficacy while limiting the risk of infection. ${ }^{24} \mathrm{~A}$ similar strategy deserves attention also in view of future vaccinations. ${ }^{25}$ However, it may not be without risks linked to the re-expansion of autoreactive $\mathrm{B}$ cells. ${ }^{26}$

The role of interferon treatment and the severity of COVID-19 is of high interest. Our data indicated a decrease of risk associated with interferon, even if it did not reach statistical significance. The majority of laboratory studies are in line with this preliminary observation, suggesting that supplementing type I interferons may circumvent a defective response that, in people with severe COVID-19, may depend on various mechanisms including the presence of anti-interferon autoantibodies, ${ }^{27}$ a 
genetically defective type I interferon response, ${ }^{28,29}$ or the ability of SARS-CoV-2 to neutralize the antiviral effects of type I interferons. ${ }^{30} \mathrm{~A}$ recent clinical trial of interferon beta- $1 \mathrm{~b}$ and lopinavir-ritonavir in Middle East respiratory syndrome ${ }^{31}$ supports this hypothesis, but a negative trial of interferon beta- $1 \mathrm{~b}$, alone or in combination with lopinavir, does not. ${ }^{32}$ It is possible that timing in the administration of type I interferons is crucial, as early therapy is associated with favorable clinical responses. ${ }^{33}$ This is in accord with our observation in patients who are already under interferon beta therapy when they develop COVID-19.

Importantly, methylprednisolone in the month preceding the first symptoms of COVID-19 was significantly associated with a worse outcome. This aspect was not considered in previous reports, is in agreement with data in other autoimmune diseases, ${ }^{34}$ and is relevant in assessing the risk of DMTs (ie, COVID-19 risk vs steroid-sparing potential).

This study has limitations that must be considered when evaluating the results. First, we included in the study not only confirmed but also suspected cases. For this reason, we cannot exclude that the suspected cases suffered from infections other than COVID-19. We took the decision of including suspected cases because, during the pandemic peak in Italy, only the most severe cases (often after hospitalization) were tested. Hence, including only confirmed cases would have limited the representativeness of our sample. The inclusion of patients suffering from non-COVID-19 infections might have diluted the effect of the detected association, resulting in a conservative approach. Furthermore, we reran all the main analyses on the subgroup of confirmed cases, obtaining similar and even stronger results, despite the smaller sample size. A second relevant bias might derive from differences in awareness and expectations of risks in patients receiving therapies with stronger immunosuppressive effect. This might have favored more frequent contacts with treating neurologists, resulting in increased attention to ocrelizumab or rituximab patients. However, the same should have been true also for other intravenous therapies. Finally, although the multivariate analysis adjusts the effect of DMTs on COVID-19 severity for the main confounding factors, we cannot exclude that some residual confounding can partly explain the observed associations.

Overall, this study shows an acceptable safety profile of DMTs in MS during the COVID-19 pandemic. The differences that emerged among the various DMTs are in agreement with previous knowledge about infections in PwMS. The results are also plausible based on current biological knowledge, although the exact mechanisms that affect the risk of COVID-19 remain uncertain. It will be important to look at other ongoing studies ${ }^{35}$ to verify whether they confirm our findings and to compare the new clinical insight in other autoimmune diseases. This may stimulate new laboratory research and shed new light on the biology of SARS-CoV-2 infection and on the pathophysiology of the different autoimmune diseases.

\section{Acknowledgments}

The Musc-19 Study Group thanks Roche for donating the web-based platform for data collection and the Department of Informatics, Bioengineering, Robotics, and Systems Engineering, University of Genoa, for its help in installing the platform.

\section{Author Contributions}

All authors contributed to the conception and design of the study; the Musc-19 Study Group contributed to the acquisition and analysis of data; M.P.S., I.S., L.C., and M.S. contributed to drafting the text and preparing the figures.

\section{Potential Conflicts of Interest}

M.P.S. reports a grant from Roche to cover data management of this study; Roche makes ocrelizumab, which is one of the DMTs assessed in this study. The remaining authors have nothing to report.

\section{References}

1. Haberman R, Axelrad J, Chen A, et al. Covid-19 in immunemediated inflammatory diseases_case series from New York. N Engl J Med 2020;383:85-88.

2. Sormani MP. Italian Study Group on COVID-19 Infection in Multiple Sclerosis. An Italian programme for COVID-19 infection in multiple sclerosis. Lancet Neurol 2020;19:481-482; erratum Lancet Neurol 2020;19:e6.

3. Louapre C, Collongues N, Stankoff B, et al. Clinical characteristics and outcomes in patients with coronavirus disease 2019 and multiple sclerosis. JAMA Neurol 2020;77:1079-1088.

4. Hughes R, Pedotti R, Koendgen H. COVID-19 in persons with multiple sclerosis treated with ocrelizumab-a pharmacovigilance case series. Mult Scler Relat Disord 2020;42:102192.

5. Browne $P$, Chandraratna D, Angood C, et al. Atlas of multiple sclerosis 2013: a growing global problem with widespread inequity. Neurology 2014;83:1022-1024.

6. Winkelmann A, Loebermann M, Reisinger EC, et al. Diseasemodifying therapies and infectious risks in multiple sclerosis. Nat Rev Neurol 2016;12:217-233.

7. Amor S, Baker D, Khoury SJ, et al. SARS-CoV-2 and multiple sclerosis: not all immune depleting DMTs are equal or bad. Ann Neurol 2020;87:794-797.

8. Battaglia MA, Bezzini D. Estimated prevalence of multiple sclerosis in Italy in 2015. Neurol Sci 2017;38:473-479.

9. Van Buuren S, Groothuis-Oudshoorn K. mice: multivariate imputation by chained equations in R. J Stat Software 2011;45:1-67. 
10. Mccaffrey DF, Griffin BA, Almirall D, et al. A tutorial on propensity score estimation for multiple treatments using generalized boosted models. Stat Med 2013;32:3388-3414.

11. Rubin EJ, Baden LR, Morrissey S. Audio interview: Lessons from Covid-19 hotspots. N Engl J Med 2020;382:e35.

12. Sahraian MA, Azimi A, Navardi $S$, et al. Evaluation of the rate of COVID-19 infection, hospitalization and death among Iranian patients with multiple sclerosis. Mult Scler Relat Disord 2020;46: 102472.

13. Safavi F, Nourbakhsh B, Azimi AR. B-cell depleting therapies may affect susceptibility to acute respiratory illness among patients with multiple sclerosis during the early COVID-19 epidemic in Iran. Mult Scler Relat Disord 2020;43:102195.

14. Parrotta E, Kister I, Charvet L, et al. COVID-19 outcomes in MS: Observational study of early experience from NYU Multiple Sclerosis Comprehensive Care Center. Neurol Neuroimmunol Neuroinflamm. 2020;7:e835. https://doi.org/10.1212/NXI.0000000000000835.

15. Montero-Escribano P, Matías-Guiu J, Gómez-Iglesias P, et al. AntiCD20 and COVID-19 in multiple sclerosis and related disorders: a case series of 60 patients from Madrid, Spain. Mult Scler Relat Disord 2020;42:102185

16. Luna $G$, Alping $P$, Burman J, et al. Infection risks among patients with multiple sclerosis treated with fingolimod, natalizumab, rituximab, and injectable therapies. JAMA Neurol 2020;77:184-191.

17. Hauser SL, Bar-Or A, Comi G, et al. Ocrelizumab versus interferon beta-1a in relapsing multiple sclerosis. N Engl J Med 2017;376: $221-234$

18. Montalban X, Hauser SL, Kappos L, et al. Ocrelizumab versus placebo in primary progressive multiple sclerosis. N Engl J Med 2017; 376:209-220.

19. Emery P, Rigby W, Tak PP, et al. Safety with ocrelizumab in rheumatoid arthritis: results from the ocrelizumab phase III program. PLoS One 2014;9:e87379.

20. Schweitzer F, Laurent S, Fink GR, et al. Effects of disease-modifying therapy on peripheral leukocytes in patients with multiple sclerosis. J Neurol 2020. https://doi.org/10.1007/s00415-019-09690-6. (Online ahead of print).

21. Ren $L$, Zhang $L$, Chang $D$, et al. The kinetics of humoral response and its relationship with the disease severity in Covid-19. Commun Biol 2020;3:780

22. Díez JM, Romero C, Vergara-Alert J, et al. Cross-neutralization activity against SARS-CoV-2 is present in currently available intravenous immunoglobulins. Immunotherapy 2020;12:1247-1255.
23. Guthmiller JJ, Wilson PC. Remembering seasonal coronaviruses. Science 2020;370:1272-1273.

24. Baker D, Pryce G, James LK, et al. The ocrelizumab phase II extension trial suggests the potential to improve the risk: benefit balance in multiple sclerosis. Mult Scler Relat Disord 2020;44:102279.

25. Bar-Or A, Calkwood JC, Chognot C, et al. Effect of ocrelizumab on vaccine responses in patients with multiple sclerosis: the VELOCE study. Neurology 2020;95:e1999-e2008.

26. Rommer PS, Milo R, Han MH, et al. Immunological aspects of approved MS therapeutics. Front Immunol 2019;10:1564.

27. Bastard P, Rosen LB, Zhang Q, et al. Autoantibodies against type I IFNs in patients with life-threatening Covid-19. Science 2020;370: eebd4585.

28. Zhang $Q$, Bastard P, Liu Z, et al. Inborn errors of type I IFN immunity in patients with life-threatening Covid-19. Science 2020;370:eebd4570.

29. Pairo-Castineira E, Clohisey S, Klaric L, et al. Genetic mechanisms of critical illness in Covid-19. Nature. 2020. https://doi.org/10.1038/ s41586-020-03065-y. (Online ahead of print).

30. Blanco-Melo D, Nilsson-Payant BE, Liu WC, et al. Imbalanced host response to SARS-CoV-2 drives development of COVID-19. Cell 2020;181:1036-1045.e9.

31. Arabi YM, Asiri AY, Assiri AM, et al. Interferon beta-1b and lopinavirritonavir for Middle East respiratory syndrome. N Engl J Med 2020; 383:1645-1656.

32. WHO Solidarity Trial Consortium, Pan H, Peto R, et al. Repurposed antiviral drugs for Covid-19-interim WHO Solidarity Trial results. N Engl J Med 2020. https://doi.org/10.1056/NEJMoa2023184. (Online ahead of print).

33. Wang N, Zhan $Y$, Zhu L, et al. Retrospective multicenter cohort study shows early interferon therapy is associated with favorable clinical responses in Covid-19 patients. Cell Host Microbe 2020;28:455-464. e2. (Online ahead of print).

34. Gianfrancesco M, Hyrich KL, Al-Adely S, et al. Characteristics associated with hospitalisation for COVID-19 in people with rheumatic disease: data from the COVID-19 Global Rheumatology Alliance physician-reported registry. Ann Rheum Dis 2020;79:859-866.

35. Peeters LM, Parciak T, Walton C, et al. COVID-19 in people with multiple sclerosis: a global data sharing initiative. Mult Scler 2020; 26:1157-1162. 